\title{
Erratum to: Lenalidomide plus dexamethasone treatment in Japanese patients with relapsed/refractory multiple myeloma
}

\author{
Shinsuke Iida $\cdot$ Takaaki Chou $\cdot$ Shinichiro Okamoto $\cdot$ Hirokazu Nagai $\cdot$ \\ Kiyohiko Hatake · Hirokazu Murakami · Toshiyuki Takagi • Kazuyuki Shimizu • \\ Henry Lau • Kenichi Takeshita · Masaaki Takatoku • Tomomitsu Hotta
}

Published online: 1 July 2010

(C) The Japanese Society of Hematology 2010

\section{Erratum to: Int J Hematol}

DOI 10.1007/s12185-010-0624-7

There are errors in Table 2. The numbers and the percentages for grades 3 and 4 adverse events are incorrectly aligned. The correct version of the table appears on the following page.

The online version of the original article can be found under doi:10.1007/s12185-010-0624-7.

\section{S. Iida $(\square)$}

Department of Medical Oncology and Immunology,

Nagoya City University Graduate School of Medical Sciences,

1, Kawasumi, Mizuho-cho, Mizuho-ku,

Nagoya 467-8601, Japan

e-mail: iida@med.nagoya-cu.ac.jp

T. Chou

Department of Internal Medicine,

Niigata Cancer Center Hospital, Niigata, Japan

\section{S. Okamoto}

Division of Hematology,

Keio University School of Medicine,

Tokyo, Japan

\section{H. Nagai · T. Hotta}

National Hospital Organization Nagoya Medical Center,

Nagoya, Japan
K. Hatake

Cancer Institute Hospital, Tokyo, Japan

H. Murakami

School of Health Sciences, Faculty of Medicine,

Gunma University, Maebashi, Japan

T. Takagi

Kimitsu Chuo Hospital, Kisarazu, Japan

K. Shimizu

Nagoya City Midori General Hospital, Nagoya, Japan

H. Lau $\cdot$ K. Takeshita

Celgene Corporation, Summit, USA

K. Takeshita $\cdot$ M. Takatoku

Celgene KK, Tokyo, Japan 
Table 2 Lenalidomide-related adverse events with NCI-

CTCAE grade 3 or $4(n=15)$

A subject with multiple occurrence of an adverse event is counted once

a In 3 cases of lymphopenia, one was reported as a lenalidomide and/or dexamethasone-related $\mathrm{AE}$

${ }^{b}$ Lenalidomide and/or dexamethasone-related AEs

\begin{tabular}{lllc}
\hline Events & Grade 3 & Grade 4 & Grade $3+4, n(\%)$ \\
\hline Patients with at least one $\leq$ grade 3 adverse event & & & $11(73.3)$ \\
Anemia & - & $2(13.3)$ & $2(13.3)$ \\
Leukopenia & $3(20.0)$ & - & $3(20.0)$ \\
Lymphopenia & $3(20.0)^{\mathrm{a}}$ & - & $3(20.0)$ \\
Neutropenia & $9(60.0)$ & $1(6.7)$ & $10(66.7)$ \\
Hypoxia & $1(6.7)$ & - & $1(6.7)$ \\
Malaise & $1(6.7)$ & - & $1(6.7)$ \\
Hepatic function abnormality $^{\mathrm{b}}$ & $1(6.7)$ & - & $1(6.7)$ \\
Decreased blood phosphorus $_{\text {Increased alanine aminotransferase }}^{\mathrm{b}}$ & $1(6.7)$ & - & $1(6.7)$ \\
\end{tabular}

